\title{
Antibiotics Susceptibility Profile of Wounds Isolates
}

Abdulbagi Saeed Elbala ${ }^{1}$, Abobaker Safe ELdeen Elkheir ${ }^{1}$, Elnaim Bushra Ahmed ${ }^{1,2}$ and Babiker Saad Almugadam ${ }^{{ }^{*}}$

${ }^{1}$ Department of Microbiology, Faculty of Medical Laboratory Sciences, University of Elimam Elmahdi, Kosti City, Sudan

${ }^{2}$ Department of Medical Laboratory Investigations, Kosti Police Hospital, Kosti city, Sudan

"Corresponding author: Almugadam BS, Department of Microbiology, Faculty of Medical Laboratory Sciences, University of Elimam Elmahdi, Kosti city, Sudan; Tel: 00249917717034; Email: Babiker888@yahoo.com

Received date: April 12, 2018; Accepted date: April 21, 2018; Published date: April 27, 2018

Copyright: @2018 Almugadam BS, et al. This is an open-access article distributed under the terms of the Creative Commons Attribution License, which permits unrestricted use, distribution, and reproduction in any medium, provided the original author and source are credited.

\begin{abstract}
Background: Wounds infection occurs due to contamination of wounds with microbes. Wounds infection can lead to serious complications as a result of localize or hematogenous spread of their causative pathogens.

Objective: This study aimed to evaluate the susceptibility of Enterobacteriaceae isolates to commonly use antibiotics for wounds infections.

Materials and Methods: This is a cross sectional, hospital and laboratory based study, carried out during period from October 2016 to August 2017. Wound swab was collected from each participant and cultured directly on blood and MacConkey agar; then incubated at $37^{\circ} \mathrm{C}$ aerobically for 24 hours. Each isolate was identified base on culture characters, Gram stain and manual biochemical tests. All isolates (hindered) which presumptively identified as a member of Enterobacteriaceae were further subjected to antimicrobial susceptibility testing (AST). Statistical analysis was performed using statistical package for social sciences (SPSS) software version 16.
\end{abstract}

Results: Antimicrobial susceptibility testing showed an emerge in antimicrobial resistant, among wounds isolates and there is significant difference in the susceptibility of this isolates to antibiotics. Most isolates were sensitive to IPM, and all were resistant to CTR $100 \%$.

Conclusions: Antimicrobial susceptibility testing will be performed as a routine test for patients with wounds infections. Further studies must focus on other highly active and cheaper alternative therapies.

Keywords: Antibiotics; Enterobacteriaceae, Sudan; Wound infection; Wounds isolates

\section{Introduction}

Wounds break the integrity of the skin and allow for organisms to gain access to tissues and cause infection [1]. Most wounds are contaminated by microbes, but always infection does not develop in most cases. A complex interplay between host immunity, microbes, and surgical factors ultimately determines the prevention or establishment of a wound infection [2]. Infections arising in surgical sites are one of the most major nosocomial infections [3]. Extracellular wounds infection is most common than intracellular infection [4].Wounds can be broadly categorized as having either an acute or a chronic etiology [5]. Infected wounds can lead to serious local and systemic complications. When the infection extended to blood stream, bacteria may spread and cause infection in other organs [6]. Wound infections occur mainly as a result of multiplication of bacteria such as Enterobacteriaceae that are family of oxidase negative, Gram negative bacilli [7]. Multidrug resistant (MDR) Enterobacteriaceae has been emerge among wounds infections worldwide which reduce the choices of selected antimicrobials therapies $[8,9,10]$. This study aimed to evaluate the susceptibility of Enterobacteriaceae isolates to commonly using antibiotics for treatment of wounds infections.

\section{Materials and Methods}

\section{Study design, area, and duration}

This observational, descriptive, cross sectional, hospital and laboratory base study, carried out in Kosti city of Sudan, during period of October 2016 to August 2017. Kosti is $317 \mathrm{~km}$ far from Khartoum to the south of Sudan (Figure 1). Study area is $39,701 \mathrm{~km}^{2}$ and their population about 1,140,694 (2008). A large number of people from Kosti locality and surrounding zones come to Kosti Teaching Hospital as outpatients to make their laboratory investigations and get their treatments.

\section{Sampling}

Only Enterobacteriaceae isolates were included in this study. All samples were collected from wounds on the base of randomize selection using cotton tipped swab under aseptic condition. During study duration a total of 100 Enterobacteriaceae ( $P$. mirabilis 33, K. pneumoniae 25, E. coli 23, E. aerogenes 11, C. freundii 8 ) were isolated from patients of different gender and ages, who came to Kosti Teaching Hospital suffering from wounds infections. 
Page 2 of 4

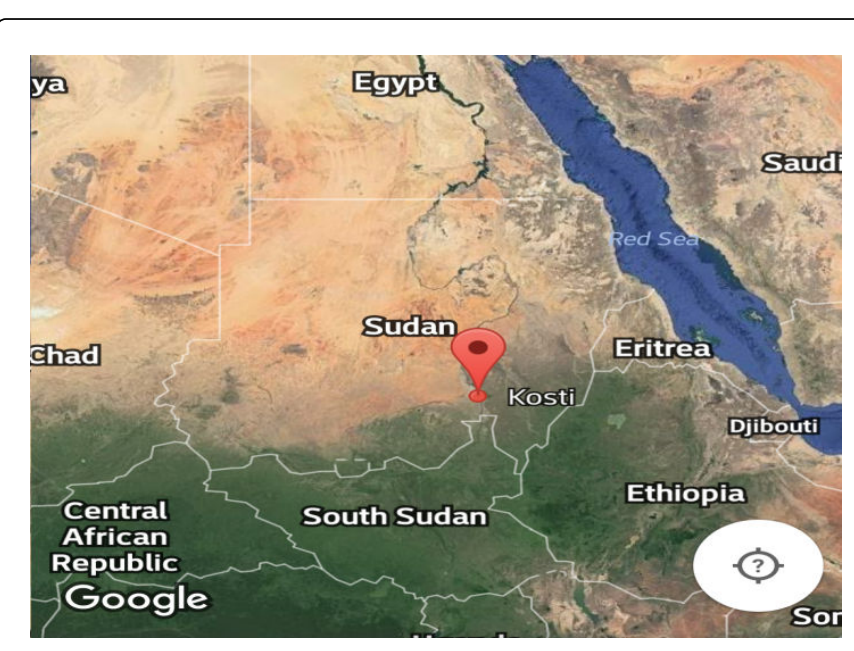

Figure 1: The location of study area (Figure was taken using Google Map, Satellite).

\section{Isolation and identification}

Wound swab were collected from each participant and cultured directly on blood and MacConkey agar and then incubated at $37^{\circ} \mathrm{C}$ aerobically for 24 hours [11]. Each isolate was identified based on culture characters, Gram stain and manual biochemical tests [12]. All isolates (hindered) which presumptively were identified as a member of Enterobacteriaceae were further subjected to antimicrobial susceptibility testing (AST).

\section{Antimicrobial susceptibility testing}

Each isolate was examined for their susceptibility to Bioanalyse

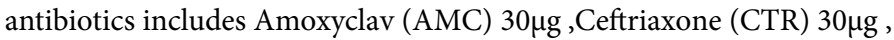

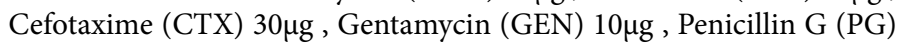
$10 \mu \mathrm{g}$, Imipenem (IPM) $10 \mu \mathrm{g}$, and Meropenem (MEM) $10 \mu \mathrm{g}$ using modified Kirby Bauer disk diffusion technique according to Clinical and Laboratory Standards Institute (CLSI) guidelines 2011 [13]. E. coli ATCC25922 strain was used as control strain.

\section{Ethical consideration}

This study was approved by department of microbiology, University of El Imam El Mahdi; and conducted according to declaration of Helsinki. Each sample was collected after he or she accepted and known that they are participate in clinical study. Verbal consent was taken from each participant.

\section{Statistical analysis}

The collected data were analyzed using statistical package for social sciences (SPSS) software version 16 and presented in form of table. One way Anova test was done and $\mathrm{p}$ value less than 0.05 were considered significant.

\section{Result}

Antimicrobial susceptibility testing showed an emerge in antimicrobial resistant among wounds isolates and there is a significant difference in the susceptibility of this isolates to most applied antibiotics. Most isolates were sensitive to IPM, and all were resistant to CTR 100\%, as we displayed in Table 1.

\begin{tabular}{|c|c|c|c|c|c|c|c|}
\hline \multicolumn{2}{|c|}{ Antibiotics } & E. coli & C. freundii & E. aerogenes & K. pneumoniae & P. mirabilis & $P$ value \\
\hline \multirow{2}{*}{ CTR } & $\mathrm{s}$ & $0 \%(0 / 23)$ & $0 \%(0 / 8)$ & $0 \%(0 / 11)$ & $0 \%(0 / 25)$ & $0 \%(0 / 33)$ & \multirow{2}{*}{-} \\
\hline & $\mathrm{R}$ & $100 \%(23 / 23)$ & $100 \%(8 / 8)$ & $100 \%(11 / 11)$ & $100 \%(25 / 25)$ & $100 \%(33 / 33)$ & \\
\hline \multirow{2}{*}{ CTX } & $\mathrm{s}$ & $4.3 \%(1 / 23)$ & $0 \%(0 / 8)$ & $0 \%(0 / 11)$ & $24 \%(6 / 25)$ & $0 \%(0 / 33)$ & \multirow{2}{*}{0.003} \\
\hline & $\mathrm{R}$ & $95.7 \%(22 / 23)$ & $100 \%(8 / 8)$ & $100 \%(11 / 11)$ & $76 \%(19 / 25)$ & $100 \%(33 / 33)$ & \\
\hline \multirow{2}{*}{ AMC } & $S$ & $34.8 \%(8 / 23)$ & $0 \%(0 / 8)$ & $100 \%(11 / 11)$ & $16 \%(4 / 25)$ & $0 \%(0 / 33)$ & \multirow{2}{*}{0.000} \\
\hline & $\mathrm{R}$ & $65.2 \%(15 / 23)$ & $100 \%(8 / 8)$ & $0 \%(0 / 11)$ & $84 \%(21 / 25)$ & $100 \%(33 / 33)$ & \\
\hline \multirow{2}{*}{ GEN } & $\mathrm{s}$ & $34.8 \%(8 / 23)$ & $100 \%(8 / 8)$ & $100 \%(11 / 11)$ & $88 \%(22 / 25)$ & $24.24 \%(8 / 33)$ & \multirow{2}{*}{0.000} \\
\hline & $\mathrm{R}$ & $65.2 \%(15 / 23)$ & $0 \%(0 / 8)$ & $0 \%(0 / 11)$ & $12 \%(3 / 25)$ & $75.76 \%(25 / 33)$ & \\
\hline \multirow{2}{*}{ PG } & $\mathrm{s}$ & $17.4 \%(4 / 23)$ & $12.5 \%(1 / 8)$ & $45.5 \%(5 / 11)$ & $12 \%(3 / 25)$ & $0 \%(0 / 33)$ & \multirow{2}{*}{0.003} \\
\hline & $\mathrm{R}$ & $82.6 \%(19 / 23)$ & $87.5 \%(7 / 8)$ & $54.5 \%(6 / 11)$ & $88 \%(22 / 25)$ & $100 \%(33 / 33)$ & \\
\hline \multirow{2}{*}{ IPM } & $\mathrm{s}$ & $95.7 \%(22 / 23)$ & $100 \%(8 / 8)$ & $100 \%(11 / 11)$ & $92 \%(23 / 25)$ & $100 \%(33 / 33)$ & \multirow{2}{*}{0.431} \\
\hline & $\mathrm{R}$ & $4.3 \%(1 / 23)$ & $0 \%(0 / 8)$ & $0 \%(0 / 11)$ & $8 \%(2 / 25)$ & $0 \%(0 / 33)$ & \\
\hline \multirow{2}{*}{ MEM } & $S$ & $87 \%(20 / 23)$ & $0 \%(0 / 8)$ & $54.5 \%(6 / 11)$ & $80 \%(20 / 25)$ & $69.7 \%(23 / 33)$ & \multirow{2}{*}{0.000} \\
\hline & $\mathrm{R}$ & $13 \%(3 / 23)$ & $100 \%(8 / 8)$ & $45.5 \%(5 / 11)$ & $20 \%(5 / 25)$ & $30.3 \%$ (10/33) & \\
\hline
\end{tabular}

Table 1: Susceptibility of Enterobacteriaceae isolates to antibiotics. 
Page 3 of 4

All C. freundii, E. aerogenes and P. mirabilis isolates were resistant to CTR and CTX and sensitive to IPM. And all E. coli isolates were resistant to CTX and sensitive to IPM and MEM.

\section{Discussion}

Wounds infection is the most critical problem especially in the presence of foreign materials that can increase the risk of complications [14]. The emergence of antibiotics resistance and its rapid spread were considered as major threats to the public health worldwide [15-17]. Our study results reveal there is a significant difference in the susceptibility of wounds isolates to antibiotics and all wounds isolates were resistant to CTR $100 \%$.

This study was found all E. coli isolates were resistant to CTR and most were resistant to CTX (95.7\%), PG (82.6\%), AMC (65.2\%) and GEN (65.2\%); while most were sensitive to IPM 95.7\%, and MEM (87\%). This result disagree with Manikandan C et al, 2013 study that reported the resistance rate of $E$. coli isolates to CTR, CTX and GEN were $37.5 \%, 87.5 \%$ and $37.5 \%$ respectively [8]. And we disagree with Mohammed A et al, 2013 study that reported the resistance rate of $E$. coli isolates to CTX and GEN was $89 \%$ for each one [18]. Also our results disagree with Bessa LJ et al, 2015 study which reported the resistance rate of E. coli isolates to CTX, MEM and GEN to be 23.5\%, $0.0 \%$ and $11.8 \%$ respectively [19].

As we displayed in Table 1 all $C$. freundii and E. aerogenes isolates were resistant to both CTR and CTX, and sensitive to GEN and IPM; while most of them were resistant to PG. Also all $C$. freundii isolates were resistant to AMC and MEM. While all E. aerogenes isolates were sensitive to AMC and most were sensitive to MEM (54.5\%). This results disagree with Mohammed A et al, 2013 study that reported the resistance rate of Citrobacter spp. isolates to CTX and GEN were 76\%, and $57 \%$ respectively and the resistance rate of Entrobacter spp. isolates to CTX and GEN were $86 \%$, and $71 \%$ respectively [18].

Our study results found all $K$. pneumoniae isolates were resistant to CTR and most were resistant to PG (88\%), AMC (84\%), and CTX (76\%). Also most $K$. pneumoniae isolates were sensitive to IPM (92\%), GEN (88\%), and MEM (80\%). This result disagree with Mohammed A et al, 2013 study that reported the resistance rate of Klebsiella spp. isolates to CTX and GEN were 56\%, and $78 \%$ respectively [18]. Also we disagree with Sultana $S$ et al, 2015 study that reported the percentage of sensitivity of Klebsiella spp. isolates to CTR, GEN, and IPM were $11.11 \%, 55.55 \%$, and $77.77 \%$ respectively [20].

As we mentioned in the results section, all $P$. mirabilis isolates were resistant to CTR, CTX, AMC and PG; and sensitive to IPM. And most were sensitive to MEM (69.7\%) and resistant to GEN (75.76\%). This result disagree with Mohammed A et al, 2013 study that reported the resistance rate of $P$. mirabilis isolates to GEN was $21 \%$ [18]. And disagree with Mohammed A et al, study in the resistance rate of $P$. mirabilis isolates to CTX which reported as $32 \%$ in Mohammed A et al, study [18]. Also we disagree with Bessa LJ et al, 2015 study which reported the resistance rate of $P$. mirabilis isolates to CTX, MEM and GEN were $42.8 \%, 3.6 \%$ and $53.6 \%$ respectively [19].

The difference in our results when compared with other studies results may arise from the difference in study population or availability of non-prescribed antibiotics in Sudan [21]. Our study found that there is a significant difference in the susceptibility of wounds isolates for all applied antibiotics except IPM and suggests IPM is only drug of value for wounds infections cause by Enterobacteriaceae and the use of combined therapy can achieve a highly synergistic affect to treat wounds infections.

\section{Conclusions}

Successful treatment of wounds infections will eliminate the pathogens and prevent the serious complications of wounds infections. Microbiological investigations are very important for selection of proper treatment especially in patients with chronic or open wounds infections. Antimicrobial susceptibility testing will be performed as a routine test for patients with wounds infections. Further studies must focus on other highly active and cheaper alternative therapies.

\section{Acknowledgements}

Authors would like to thanks participant patients, and staff of Kosti Teaching Hospital for their support during samples collection.

\section{References}

1. Facility guidance for control of carbapenem-resistant Enterobacteriaceae (CRE)-November 2015 Update CRE Toolkit (2015) Centre for Disease Control and Prevention 1: 1-20.

2. Muhammad MH, Swedan S (2015) Molecular and phenotypic characterization of carbapenem resistance and extended spectrum betalactamases among urinary tract E.coli isolates. Int J Sci Technol 5: 1-14.

3. Sidjabat H, Nimmo GR, Walsh TR, Binotto E, Htin A, et al. (2011) Carbapenem resistance in Klebsiella pneumoniae due to the New Delhi Metallo-ß-lactamase. Clin Infect Dis 52: 481-484.

4. Brenner BM (2008) Brenner and Rector's the kidney. Philadelphia: Saunders Elsevier USA.

5. Jacoby GA, Munoz-price LS (2005) The new beta-lactemases. N Engl J Med 352: 380-391.

6. Hidron Al, Edwards JR, Patel J, Horan TC, Sievert DM, et al. (2008) NHSN annual update: Antimicrobial-resistant pathogens associated with healthcare-associated infections: Annual summary of data reported to the national healthcare safety network at the centers for disease control and prevention, 2006-2007. Infect Control Hosp Epidemiol 29: 996-1011.

7. Kiremitci A, Dinleyici EC, Erben N, Durmaz G, Yargic ZA, et al. (2008) In vitro activity of ertapenem and other carbapenems against extendedspectrum beta-lactamse producing $E$. coli and $K$. pneumoniae clinical isolates in a tertiary care center in Turkey. Expert Opin Pharmacother 9: 1441-1449.

8. Manikandan C, Amsath A (2013) Antibiotic susceptibility of bacterial strains isolated from wound infection patients in Pattukkottai, Tamilnadu, India. Int J Curr Microbiol App Sci 2: 195-203.

9. Mahat P, Manandhar S, Baidya R (2017) Bacteriological profile of wound infection and antibiotic susceptibility pattern of the isolates. J Microbiol Exp 4: 1-8.

10. Farrag HA, El-Rehim HA, Hazaa MM, El-Sayed SAS (2016) Prevalence of pathogenic bacterial isolates infecting wounds and their antibiotic sensitivity. J Infec Dis Treat 2: 1-7.

11. Bae IK, Kang HK, Jang IH, Lee W, Kim K, et al. (2015) Detection of carbap- enemases in clinical Enterobacteriaceae isolates using the VITEK AST-N202 card. Infect Chemother 47: 167-174.

12. Koneman EW, Allens SO, Janda WM, Schreck-enberger PC, Winn WC (2006) Colour atlas and textbook for diagnostic microbiology. Philadelphia: JB Lippincott Company. 4th Edition.

13. Performance standards for antimicrobial susceptibility testing; 21st informational supplement (2011) Clinical and Laboratory Standards Institute 31: 1-165.

14. Rubin RH (2006) Surgical wound infection: Epidemiology, pathogenesis, diagnosis and management. BMC Infect Dis 6: 171 . 
Citation: Elbala AS, Elkheir ASE, Ahmed EB, Almugadam BS (2018) Antibiotics Susceptibiltiy Profile of Wounds Isolates. J Antimicrob Agents 4: 169. doi:10.4172/2472-1212.1000169

Page 4 of 4

15. Rice LB (2006) Antimicrobial resistance in Gram-positive bacteria American J Med 119: S11-S19.

16. Misic AM, Gardner SE, Grice EA (2014) The wound microbiome: Modern approaches to examining the role of microorganisms in impaired chronic wound healing. Adv Wound Care 3: 502-510.

17. Iredell J, Brown J, Tagg K (2016) Antibiotic resistance in Enterobacteriaceae: Mechanisms and clinical implications. BMJ 352: h6420.

18. Mohammed A, Adeshina GO, Ibrahim YK (2013) Incidence and antibiotic susceptibility pattern of bacterial isolates from wound infections in a tertiary hospital in Nigeria. Trop J Pharm Res 12: 617-621.
19. Bessa LJ, Fazii P, Di Giulio M, Cellini L (2015) Bacterial isolates from infected wounds and their antibiotic susceptibility pattern: Some remarks about wound infection. Int Wound J 12: 47-52.

20. Sultana S, Mawla N, Kawser S, Akhtar N, Ali MK (2015) Curent microbial isolates from wound swab and their susceptibility pattern in a private medical college hospital in Dhaka city. Delta Med Col J 3: 25-30.

21. Awad A, Eltayeb I, Matowe L, Thalib L (2005) Self-medication with antibiotics and antimalarials in the community of Khartoum State, Sudan. J Pharm Pharmaceut Sci 8: 326-331. 